rheuma plus $2017 \cdot 16: 113$

https://doi.org/10.1007/s12688-017-0147-x

C Springer-Verlag GmbH Austria 2017

\section{Burkhard Leeb}

II. Medizinische Abteilung, NOE Kompetenzzentrum für Rheumatologie, Stockerau

\title{
Wie geht es weiter?
}

\section{Liebe Leserinnen und Leser!}

auch dieses Jahr folgen wir der Tradition, dass die Nummer 4 von Rheuma plus um die Zeit der Jahrestagung der Österreichischen Gesellschaft für Rheumatologie und Rehabilitation (ÖGR) erscheint. 2017 hat einige, vor allem legistische und sozialversicherungstechnische, Veränderungen mit sich gebracht, deren Auswirkungen noch nicht völlig abzusehen sein werden.

Wie wird sich etwa die Änderung der Ausbildungsordnung, die ja eigentlich nur den bevorstehenden Medizinermangel zu kupieren versucht, auf die Versorgungssituation und damit auf die Behandlungsqualität der Patienten mit rheumatischen Erkrankungen auswirken? Wird das Interesse an der Ausbildung zum Facharzt für Innere Medizin/Rheumatologie groß genug sein, um den Bedarf zu decken, der ja derzeit kaum zufriedenstellend erfüllt wird? Schon jetzt klafft eine deutliche Lücke zwischen der rheumatologischen Versorgung im stadtnahen und -ferneren Bereich, ist für viele Betroffene die Distanz zum nächsten Rheumatologen relativ groß. Die ÖGR jedenfalls unternimmt redliche Anstrengungen, die in einer Summer School für Studierende gipfelten, welche, wie man hört, sehr gut angenommen wurde.

Wird das Gesundheitssystem es sich nach wie vor leisten können, eine eigentlich ambulante Disziplin wie die Rheumatologie hauptsächlich in Spitalsambulanzen anzusiedeln? Und das nur deshalb, weil es keine tragfähigen Abrechnungsmodelle gibt, die eine Niederlassung als Rheumatologe mit Kassenverträgen ohne unmittelbare Gefahr auf Bankrott erlauben; vor allem weil die klinische Untersuchung nicht ausreichend honoriert wird. Diese klinische Untersuchung ist es aber, welche die rheumatologische Diagnostik und damit das Schicksal des Patienten bestimmt und nicht die Apparatemedizin bzw. die von reiner Ökonomie geleiteten Standardisierungsmanien, die das patientenbezogene ärztliche Handeln auszuhöhlen versuchen.

\section{Biologika, Biosimilars, Small Molecules}

Sicherlich mit Qualität der Patientenbetreuung zu tun hat der Beitrag von Edith Wipfler-Freißmuth, Graz, die über die rasante Entwicklung neuer Substanzen in der Rheumatologie berichtet. Nach TNF$a$-Inhibitoren kristallisieren sich Interleukin-Hemmer und Small Molecules wie JAK-Inhibitoren als neue Therapieoptionen - einer ab Anfang November in der gelben Box - heraus. Von großem Interesse wird es sein, zu sehen, wie dieser Wettkampf zwischen Therapieprinzipien, Darreichungsformen und Ökonomie ausgehen wird. In der eigentlich gesättigten österreichischen Biologika-Landschaft wird das ökonomische Ergebnis der Biosimilars hochgradig vom nicht-medizinisch indizierten Switch abhängen.

Rheuma plus hat in den letzten Ausgaben versucht, mit einigen Fallberichten den Fokus auf die Problemstellungen der klinischen Rheumatologie zu legen und damit auch die Vielgestaltigkeit rheumatologischer Symptomatik darzustellen. In Fortsetzung dieser Linie berichtet Nicole Aringer, Stockerau, über eine primär als kardiologisch eingestufte Symptomatik im Rahmen einer Biologikatherapie, die sich bei näherer Betrachtung als rheumatologische Erkrankung herausstellte.

Markus Köstenberger, Klagenfurt, beschäftigt sich in diesem Zusammenhang mit Opiaten als unverzichtbarem Bestandteil der Schmerztherapie nach der Durchführung einer systematisierten Schmerzerfassung. Ziel sollte es immer sein, die körperliche und geistige sowie soziale Funktionsfähigkeit unserer Patienten zu erhalten.
Neben Medikamenten und Trainingsbehandlung stellt auch die physikalische Therapie eine wesentliche Säule eines erfolgreichen Therapiekonzeptes rheumatischer Erkrankungen dar. Elisabeth Preisinger, Wien-Hietzing, gibt einen Überblick über Wärme-und Kältetherapie, wobei sie ganz allgemein bei akuten Entzündungen, neurogener Spastik, nach Traumen oder Verbrennungen, Kryotherapie indiziert, zur Besserung der Gelenksteifigkeit und Dehnbarkeit des Bindegewebes Wärmetherapie empfiehlt.

Auch mit dieser Ausgabe hoffen wir wieder, Ihr Interesse zu wecken und dem Ziel von rheuma plus nahe zu kommen, eine fruchtbringende, offene und redliche Diskussion in Gang zu bringen, aus der wir alle Nutzen ziehen können. Wie immer an dieser Stelle möchten wir Sie herzlich dazu einladen, uns Ihre Meinung zu den Beiträgen dieser Ausgabe, wie auch $\mathrm{zu}$ allen rheumatologischen Themen, die Sie für relevant oder für diskussionswert halten, zu schicken. Sie können in jedem Falle auf einen fairen Review mit entsprechendem Feedback vertrauen. Für Kritik, Hinweise, Zustimmung wie auch für jeden anderen Beitrag bedanke ich mich schon im Voraus.

\section{Herzlichst,}

Ihr Burkhard Leeb

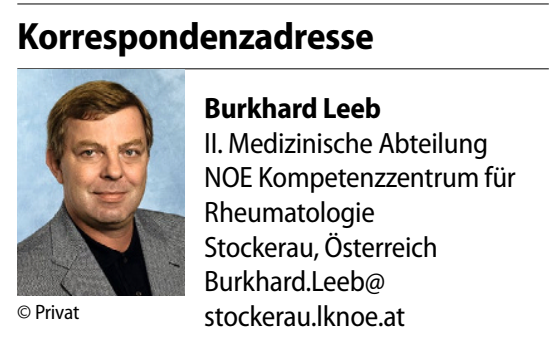

